\title{
Colitis and Colorectal Cancer
}

\author{
Sebastian Foersch Maximilian J. Waldner Markus F. Neurath \\ Department of Medicine I, Friedrich Alexander University, Erlangen, Germany
}

\section{Key Words}

Inflammatory bowel disease $\cdot$ Colitis-associated

carcinoma $\cdot$ Colorectal cancer

\begin{abstract}
Inflammatory bowel diseases (IBD) are accompanied by an increased risk of developing colitis-associated carcinoma (CAC). These tumors are one of the most important causes of morbidity and mortality in patients with IBD and distinctly differ from sporadic colorectal cancer in their biology and underlying mechanisms. First, this review discusses risk factors for the development of CAC and summarizes some of the most important genetic alterations and molecular pathways involved in inflammatory carcinogenesis. Then, new endoscopic techniques, such as chromoendoscopy and confocal laser endomicroscopy, and their contribution to surveillance and early detection of CAC are presented. Last, we briefly address different types of concepts for prevention (i.e. anti-inflammatory agents) and treatment (i.e. surgical resection) of CAC and give an outlook on this important aspect of IBD.

Copyright $\odot 2012$ S. Karger AG, Basel
\end{abstract}

\section{Introduction}

First reports about inflammatory bowel diseases (IBD) - especially the two subtypes denoted ulcerative colitis (UC) and Crohn's disease (CD) - became frequent in the beginning of the 20th century. Since then, UC and $\mathrm{CD}$ have stepped into the focus of gastroenterologic re-
(C) 2012 S. Karger AG, Basel

0257-2753/12/0305-0469\$38.00/0

Fax +41613061234 E-Mail karger@karger.ch www.karger.com
Accessible online at: www.karger.com/ddi search and practice [1]. Already in their early case studies, Crohn and Rosenberg [2] described the association of IBD with colorectal cancer (CRC). Today it is well established that UC and CD [3, 4] pose a high risk of developing colonic adenocarcinoma, especially if colitis is diagnosed at a young age and is accompanied by long disease duration, right-sided colitis, pancolitis [5], and/or primary sclerosing cholangitis [6]. In fact, chronic colitis together with familiar adenomatosis and Lynch syndrome are the three high-risk conditions, although they account for only a small portion of CRC cases. Vice versa, colitisassociated carcinoma (CAC) is one of the major causes of morbidity and mortality in patients with UC. In a large meta-analysis, Eaden et al. [7] estimated the cumulative risk of CAC to be $1.6 \%$ at 10 years, $8.3 \%$ at 20 years, and $18.4 \%$ at 30 years. As Lashner et al. [8] and Velayos et al. [9] reported, further contributing factors are colonic strictures and postinflammatory polyps, but interestingly proctitis and proctosigmoiditis do not increase the hazard of developing colonic neoplasia [10]. Also the use of pharmacologic therapy to treat IBD and maintain remission is speculated to modulate the risk of developing dysplasia/neoplasia and will be discussed in further detail in the section dealing with the treatment of CAC.

\section{Pathogenesis}

Extensive research has been carried out dealing with the underlying pathophysiology of the development and progression of sporadic CRC. The generally accepted 'hypothesis' by Vogelstein et al. [11] describes a multistep 
process from normal gastrointestinal epithelium via hyperproliferation and adenomatous polyps to an invasive carcinoma. But, while in sporadic cancer genetic disposition is a key player that drives carcinogenesis, in CAC inflammation is regarded as the deciding factor. Genetics is not completely off the table in CAC as Nuako et al. [12] were able to show that patients with IBD and a familiar history of gastrointestinal tumors have a two-fold risk increase of developing CAC compared to patients with IBD only. However, the constant inflammatory stimulus leads to lesions of completely different morphologic and biologic compositions with flat, serrated, and importantly often times multifocal polypous structures. Numerous mutations that can be found in sporadic CRC can also be observed in CAC, but at different time points in carcinogenesis and with different underlying etiologies (fig. 1).

Mutations in the APC gene are usually regarded to be the initiating step in sporadic colorectal carcinogenesis [13]. However, in UC mucosa they are only rarely observed and (if at all) play a role only in late dysplasia carcinoma progression [14]. For example, Fogt et al. [15] were able to show no loss of heterozygosity $(\mathrm{LOH})$ in the APC gene loci in normal UC mucosa (0/6) or chronic colitis $(0 / 7)$. Twenty-seven percent (3/11) of cases in high-grade dysplasia and 50\% (3/6) of cases in carcinoma showed APC mutations. In contrast, $21 \%(4 / 19)$ of APC mutations were apparent in sporadic colon adenomas.

For the p53 tumor suppressor gene it is well established that gene mutations and $\mathrm{LOH}$ appear at early time points in the inflammation-low-grade/high-grade dysplasiacarcinoma sequence in IBD - a direct contrast to the molecule's role in sporadic CRC where it is regarded as the one of the last crucial protectors from invasive carcinoma. p53 deletion was observed in about $50-85 \%$ of colitis-associated cancers [16]. $\mathrm{LOH}$ also seems to correlate with malignant progression. It was detected in $6 \%$ of colitis mucosa specimens, $33 \%$ of low-grade dysplasia specimens, $63 \%$ of high-grade dysplasia specimens, and $85 \%$ of CAC specimens. Hussain et al. [17] even observed over $50 \%$ of p53 mutations in samples of patients with active UC without any signs of neoplasm. In clearer detail, Brentnall et al. [18] described in carefully mapped colectomy specimens that p53 mutations succeed aneuploidy, which is then followed by $\mathrm{LOH}$ in the p53 gene. One major mechanism that links inflammation with p53 alterations is oxidative stress induced by the innate immune cells via reactive oxygen and nitrogen species $[19,20]$. Oxidative stress was also observed to impair DNA repair mechanisms resulting in microsatellite instability, another frequent cancer predisposition found in inflamed mucosal tissue [21]. Further steps in 'inflammatory carcinogenesis' in UC are the loss of tumor suppressor gene DCC resulting in low-grade dysplasia followed by the activation of proto-oncogenes, i.e. src and k-ras in high-grade dysplasia [22].

Apart from genomic alterations and oxidative stress, inflammatory cy tokines, other chemokines, and inflammatory transcription factors seem to play an important role in CAC development - at least in animal experiments after induction of colitis with DSS or CAC with AOM/ DSS.

For example, NF- $\kappa \mathrm{B}$, one key regulator of inflammation, and activation is found in many solid tumors and cancer cell lines [23]. Greten et al. [24] studied the role of the NF- $\kappa \mathrm{B}$ pathway in a mouse model for UC-associated CRC by knocking down the IK $\beta$ kinase, an important enzyme upstream of NF- $\kappa$ B. Disruption of the IKK $\beta$ pathway led to reduction of the tumors in size and number. It was then shown that Toll-like receptors play an important role in the interplay between the intestinal microflora and the mucosal immune defense supposedly also via NF- $\kappa B$ activation [25].

Also activation of inflammatory transcription factors leads to secretion of interleukin (IL)- 6 by T cells which has been shown to promote tumor growth via signal transducers and activators of transcription (STAT) [26]. Supporting these interesting data, high levels of IL-6 could be found in patients with colon carcinoma [27]. Tumor necrosis factor (TNF), which is secreted by macrophages as well as T cells and acts through TNF receptor-1, plays a prominent role in IBD. In fact, TNF-targeting drugs are an inherent part of the anti-inflammatory regime in CD as well as UC. TNF also plays a role in carcinogenesis by inducing DNA damage and promoting angiogenesis. TNF-R1-deficient mice that were exposed to AOM/DSS had reduced inflammation and showed fewer colitis-associated tumors. Additionally, bone marrow chimera from TNF-R1-deficient mice or mice treated with etanercept, a soluble TNF receptor, developed fewer colonic neoplasms [28]. This is coherent with newer data in mice receiving long-term DSS mimicking chronic colitis, which were treated with infliximab. Seventy-five to eighty percent of control mice developed tumors, compared to only $16.7 \%$ of mice treated with the anti-TNF agent. Interestingly, only treatment at early time-points of DSS colitis prevented the animals from developing carcinogenesis. This supports the idea of a 'top-down' treatment rather than a 'step-up' regimen [29]. Unfortunately, to date there are no good data dealing with a potential effect on colitis-associated carcinogenesis in patients receiving anti-TNF treatment. 
Fig. 1. Diagram of inflammatory carcinogenesis in colitis-associated cancer.

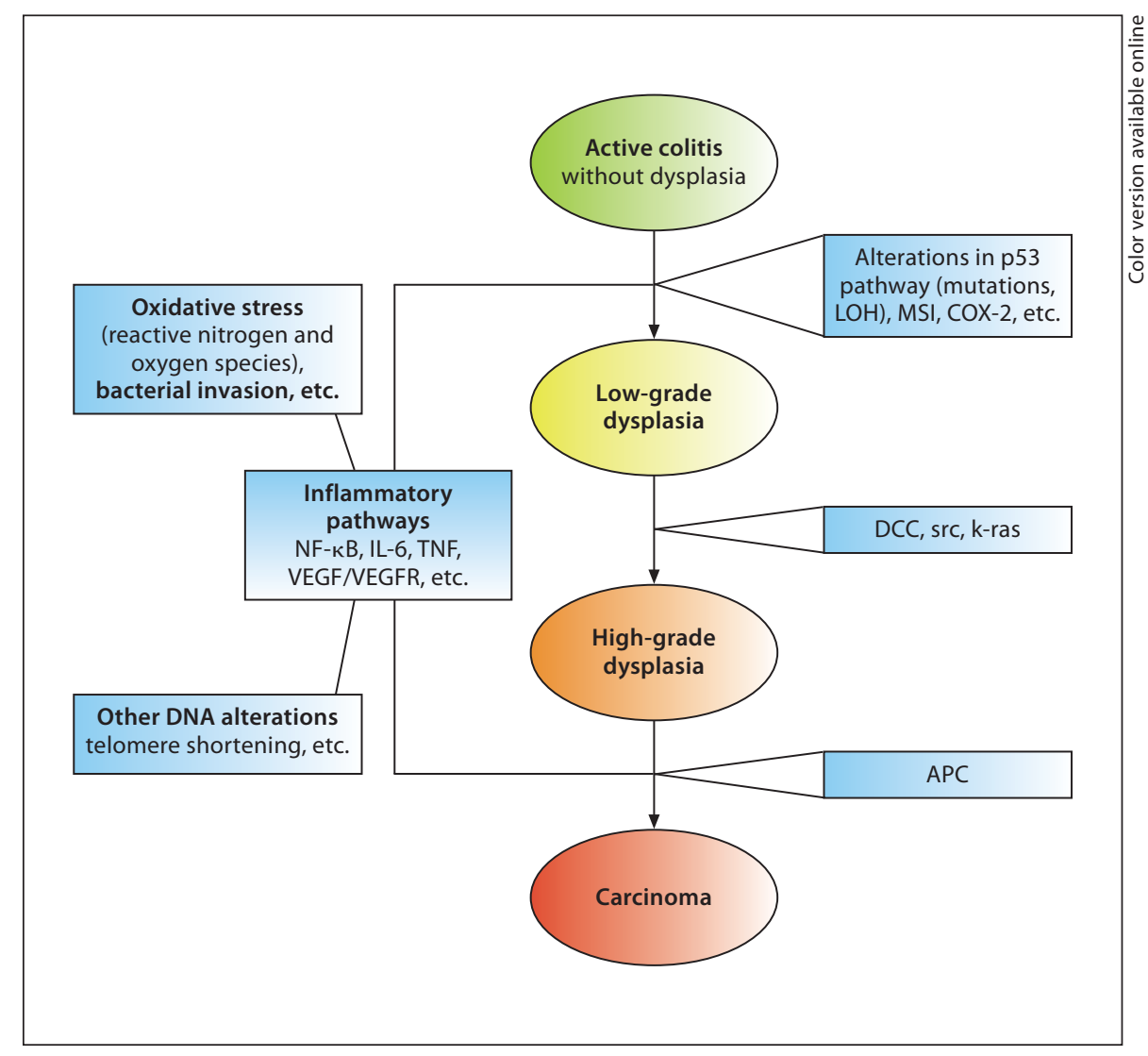

While in sporadic CRC angiogenesis and VEGF/ VEGFR signaling were studied extensively, in colitis and CAC this aspect has only recently been tapped. In sporadic CRC it was found that VEGF plays a crucial role in tumor development, and inhibition of this growth factor increased the overall survival in a large phase III trial [30]. Apart from tumor development, growing evidence supports a role for angiogenesis in the pathogenesis of IBD [31]. VEGF is secreted by various immune cells at the site of inflammation to induce angiogenesis and also directly activate other cells of the immune system [32]. Scaldaferri et al. [33] were able to show that VEGF-A induces angiogenesis of human intestinal microvascular endothelial cells in vitro. Furthermore, DSS colitis was aggravated in mice overexpressing VEGF-A, whereas overexpression of soluble VEGFR-1 had the opposite effect. It was then shown that VEGFR-2 signaling links inflammation and CAC in a STAT3-dependent manner. Interestingly, in their thoroughly conducted trial, Waldner et al. [34] were not able to show that VEGFR-2 blockage had a positive effect on the colitis itself, highlighting its role in carcinogenesis.

Colitis and Colorectal Cancer
Another finding provides an interesting but also controversial explanation for the importance of inflammation in colorectal carcinogenesis. Aspirin and nonsteroidal anti-inflammatory drugs (NSAIDs - i.e. ibuprofen) were found to decrease the risk of developing sporadic CRC [35]. Additionally, they even have a positive effect on the prevention of neoplasms in familiar adenomatous polyposis, a congenital genetic disorder [36]. This conclusion was further supported by a systematic review and meta-analysis of the literature by Velayos et al. [37] showing that anti-inflammatory agent 5-aminosalicylic acid can lower the risk of CRC in patients with IBD, although the mechanisms underlying this chemopreventive effect of anti-inflammatory agents remain to be fully elucidated. In contrast, a new study by Ishikawa and Herschman [38] found that tumor formation does not require COX-1 or COX-2 expression in AOM/DSS-treated mice. Interestingly, after repeated AOM injections without an inflammatory stimulus by DSS, COX knockout mice were somewhat protected against carcinogenesis. Thus, further studies concerning the role of COX and the effect of NSAIDs are needed. 


\section{Diagnosis}

Given the increased risk of patients with UC and CD for CRC, surveillance colonoscopy is considered to be the gold standard in diagnosing intraepithelial neoplasia in IBD patients, although the role of these preventive programs remains controversial [39]. In a retrospective study in 600 patients in a colonoscopic surveillance program over 30 years, 131 colonoscopies had to be performed for 1 beneficial procedure. Although some patients, especially those with early asymptomatic cancers, showed a benefit, surveillance was far from perfect, with 13 patients developing interval cancers during the program [40]. Additionally, older data indicates that in $50-80 \%$ of cases of CAC lesions are not visible during colonoscopy [41]. This highlights the role of another procedure, which is not completely free of risks: multiple nontargeted biopsies. Rubin et al. [42] calculated that at least 56 nontargeted biopsies need to be taken at every single surveillance colonoscopy in order to rule out dysplasia with a $95 \%$ confidence interval. This is far from optimal, does not include processing and sampling errors, and highlights the urgent need for new and advanced endoscopic techniques and procedures.

However, before looking at the new developments in endoscopic diagnostic tools, it is important to understand the terminology of malignant lesions and their precursors in IBD. Intraepithelial neoplasia is the updated term for dysplasia and subsumes all precancerous lesions in IBD. When characterized macroscopically during endoscopy, dysplasia may be either flat or raised, either local (unifocal) or multifocal. Additionally, dysplasia is further divided into low-grade dysplasia and high-grade dysplasia. The term dysplasia-associated lesion or mass (DALM) was originally applied to dysplastic lesions seen during endoscopy, which cannot be removed [43]. DALMs can present as flat, sessile, irregular, or ulcerated. The term ALMs refers to lesions which appear similar to sporadic adenomas and are amendable for resection. Especially in highly inflamed mucosal tissue, epithelial repair and regeneration processes can mimic atypia, which is difficult to distinguish from true dysplasia. This condition is denoted 'indefinite for dysplasia' [44].

In recent years, new emerging endoscopic imaging techniques were introduced, allowing a detailed analysis of mucosal and submucosal features. Similar to the entertainment industry, the use of high-definition technology during endoscopy allows higher resolutions and up to 150 -fold magnification of mucosal structures. New highpixel density CCD chips can improve the minimal dis- tance between pixels that can be resolved. Hoffman et al. [45] showed that HD endoscopy substantially helped to recognize and characterize flat neoplastic changes.

Another technique called chromoendoscopy utilizes different colored dyes, which are typically applied topically via a spraying catheter to enhance mucosal structures and patterns. These so-called pit patterns have been well described by Kudo et al. [46] (Kudo pit pattern classification) and the appearance of the crypt helps to differentiate neoplastic from nonneoplastic changes and enables the performance of targeted biopsies. The dyes used in chromoendoscopy, which is a long-established procedure, can be divided into absorbable agents (i.e. Lugol's stain and methylene blue), contrasting agents (i.e. indigo carmine and acetic acid), and agents with color reaction (i.e. Congo red and phenol red) as recently done by Neumann et al. [47]. The 'renaissance' of chromoendoscopy came when Kiesslich et al. [48] found that the technique leads to a more accurate diagnosis of the degree and extent of inflammatory activity in UC patients compared with conventional colonoscopy in a randomized, controlled study. Furthermore, chromoendoscopy significantly improved early detection of intraepithelial neoplasia and CAC in patients with UC. Another study with back-to-back colonoscopy examined pancolonic chromoendoscopy with $0.1 \%$ indigo carmine for the detection of CAC and precursors in UC. Nontargeted biopsies detected no dysplasia in almost 3,000 biopsies, while the procedure after pancolonic staining required fewer biopsies and detected 9 sites of dysplasia [49]. A meta-analysis of 6 randomized controlled trials evaluated the diagnostic accuracy of chromoendoscopy for CAC detection and found a medium/ high sensitivity and a high accuracy for diagnosis of dysplastic lesions [50].

Other techniques also highlight different mucosal features, but they use additional filters and computeral algorithms prior to or post image acquisition. This is called digital- or dye-less chromoendoscopy. Three major systems are on the market today: narrow band imaging, which utilizes an optic phenomena, i.e. the infiltration depth of light into the tissue depends on the light's wavelength. By using rotating mirrors only two wavelengths are permitted to infiltrate the mucosa: 415 and $540 \mathrm{~nm}$. Light $415 \mathrm{~nm}$ in wavelength will be absorbed by the upper mucosal layers, while longer $540-\mathrm{nm}$ waves will reach deeper and are selectively absorbed by the hemoglobin within the erythrocytes inside the submucosal vessels. The two alternative systems denoted i-scan and FICE use a digital postacquisition algorithm for computed spectral 
estimation to achieve a better tissue contrast and enhance certain mucosal features.

Despite reports showing that these three techniques can support the endoscopist, good data on the superiority of dye-less chromoendoscopy is rare. Only recently a study proved that NBI seems to be at least equally effective but faster than chromoendoscopy for the detection of intraepithelial neoplasia in IBD patients [51]. Although NBI showed significantly fewer false-positive biopsies, miss rates were still high and NBI is not recommended for standard surveillance procedures to date. In a recent prospective, randomized, controlled trial the Mainz group showed that HD colonoscopy with i-scan is superior to standard video colonoscopy in detecting colorectal neoplasia, but no special interest was paid to the special circumstances in IBD patients [52].

Confocal laser endomicroscopy (CLE) is a novel diagnostic tool which has changed modern endoscopy. CLE uses a new generation of miniaturized confocal microscopic probes that are either integrated into the tip of conventional endoscopes or can be inserted and used through the working channel. Thus, they provide the endoscopist with direct in vivo histologic images of the mucosa during ongoing endoscopy. Different fluorescent dyes have to be used either systemically or topically to provide tissue contrast. The resolution that can be achieved by bringing the confocal probe in close contact with the mucosal tissue reaches from cellular to even subcellular structures, which resembles a $1,000 \times$ magnification of a tissue portion about the size of a quarter of a square millimeter. Since the first landmark trial by Kiesslich [53] in 2004, various studies have evaluated the technique's potential in different fields of research. For precancerous lesions in IBD, again Kiesslich et al. [54] were able to show that a combination of chromoendoscopy and CLE could detect 4.75 -fold more neoplasias in surveillance colonoscopies of patients with UC compared to conventional endoscopy. Further, 50\% fewer biopsies were necessary and CLE could predict neoplastic changes with a sensitivity of $95 \%$, a specificity of $98 \%$, and an accuracy of about 98\%. Also CLE was feasible to differentiate DALMs and ALMs, with a high accuracy of $97 \%$ and an excellent correlation between CLE and the gold standard: histological diagnosis $(\kappa=0.91)$ [55]. Only recently, CLE was even used to perform molecular-targeted confocal imaging in vivo in various models of sporadic CRC. This was achieved by targeting different tumor epitopes such as VEGF [56] and EGFR [57] with fluorescently labeled antibodies. Even precursor lesions such as aberrant crypt foci could be visualized specifically by detecting CD44v6 in endo- scopic biopsy specimens [58]. Unfortunately, the potential of molecular-targeted CLE has not been explored in IBD and colitis-associated cancer yet.

In summary, other than in sporadic CRC, the role of surveillance colonoscopy and its timing remains controversial. There are multiple new high-end endoscopic imaging techniques - some of which could truly improve the diagnosis of CAC and its precursors. The problem remains that most of these techniques are only available in large endoscopy centers and are not suitable for widespread screening. Furthermore, more studies have to be conducted to evaluate whether these costly technologies really yield an additional benefit.

\section{Therapy}

There are two aspects in the therapy of CAC, this review focuses on: (i) the role of pharmacologic treatment in the development of neoplastic lesions in IBD patients and (ii) the role of surgical intervention (most likely colectomy) as a curative treatment.

For sporadic carcinoma it is well established that pharmacologic prevention can reduce the patient's risk and lower incidence. As described, the most effective chemopreventive agents for CRC are drugs inhibiting inflammation (COX inhibitors and other NSAIDs), a hint that sporadic CRC and inflammation-associated cancer are somehow promoted by similar pathways. Since inflammation is also seen as the major stimulus for CAC in IBD patients, there is a strong rationale for the use of antiinflammatory treatment as a prevention strategy. While several studies support this concept, the evidence is somehow conflicting due to differences in study design and details of the medication regime.

For example, aminosalicylates are inexpensive, safe, and already part of the maintenance therapy in IBD patients with long-standing inflammation. A number of studies have been conducted to evaluate the potential of mesalazine and sulfasalazine for chemoprevention of CAC. For example, Eaden et al. [59] were able to show a reduction in the incidence of CAC of over $75 \%$ using mesalazine. Although sulfasalzine was not as effective, both drugs showed a dose-dependent effect. The biggest risk reduction was observed when $>1.2 \mathrm{~g} /$ day were administered, even after adjusting for other variables such as age and disease duration. However, while several other studies showed similar results, some groups were not able to reproduce these findings and could not observe any significant effect. For example, Lindberg et al. [60] performed a retrospective 
analysis of the use of sulfasalazine or mesalamine treatment, and although there was a slightly lower rate of dysplasia and CAC among treated patients compared with nontreated patients, this was not statistically significant. However, even though some studies have not shown a statistically relevant difference, the majority of the investigations have found at least a protecting effect, supporting the role of mesalazine and to some extent sulfasalazine as chemopreventive agents in CAC development.

Other studies, mostly in patients with UC and primary sclerosing cholangitis, have evaluated the effect of ursodesoxycholic acid, administered to treat liver disease. It could be observed that patients receiving treatment had a lower incidence of CAC and dysplasia compared to untreated patients $[61,62]$. The underlying mechanism of this is somehow unclear, but it is suggested that ursodesoxycholic acid has an antioxidant potential and carcinogenic bile acids are reduced [63]. Recent data shows the role of ursodesoxycholic acid differently, as Eaton et al. [64] observed an increase in tumor incidence in patients receiving high doses of the drug. Unfortunately, to date there is no study evaluating the role of therapeutic antibodies in the prevention of dysplasia and CAC. A hint can be found in a report by Caspersen et al. [65], who performed a retrospective study on follow-up data of patients treated with infliximab. Of 651 patients, 4 developed CAC whereas 5.9 patients were expected to, calculated from epidemiologic data.

As described above, current guidelines for $\mathrm{UC}$ and $\mathrm{CD}$ recommend the first surveillance endoscopy 8-10 years after onset of the first symptoms and it should be repeated every 1-2 years for 20 years. Patients with PSC should be screened annually. Guidelines also define when patients should undergo preventative pancolectomy: if, after surveillance colonoscopy, targeted or nontargeted biopsy specimens reveal a flat high-grade dysplasia, which is confirmed by an expert on GI pathology, colectomy is indicated. If the high-grade dysplasia is raised and it can be confirmed that it was removed completely, colonos- copy should be repeated in 6-month intervals to confirm the absence of further dysplasia. The same pattern should be applied for raised and flat low-grade dysplasia. Indefinite findings for dysplasia should result in repeated colonoscopy within 3-6 months (probably positive) or 6-12 months (probably negative), respectively. If colonoscopy reveals a DALM, it should be clarified if it is an adenomalike DALM or a nonadenoma-like DALM. The former should result in polypectomy with increased surveillance if the lesion resides inside colitis. The latter should result in colectomy [66].

\section{Conclusion}

In summary, colitis-associated neoplasia is - for good reason - one of the most dreaded complications in patients with IBD. Although extensive research has been carried out and good data could be obtained from drawing parallels to sporadic CRC, the pathogenesis of 'inflammatory carcinogenesis' is far from being completely understood [67]. We are only beginning to realize the enormous importance of our immune system [68] and inflammation in the development of colorectal carcinoma and many other kinds of tumors.

Diagnosis of CAC is one of the big challenges in modern gastrointestinal endoscopy and might be greatly improved by novel imaging and endoscopic resection techniques. Detailed guidelines provide clear instructions and should be thoroughly adopted and applied in clinical practice to minimize the risk of tumor development for the IBD patient $[69,70]$.

\section{Disclosure Statement}

The authors declare that no financial or other conflict of interest exists in relation to the content of the article.

\section{References}

1 Kirsner JB: Historical aspects of inflammatory bowel disease. J Clin Gastroenterol 1988; 10:286-297.

- 2 Crohn B, Rosenberg H: The sigmoidoscopic picture of chronic ulcerative colitis (nonspecific). Am J Med Sci 1925;170:220-228.

- 3 Jess T, Gamborg M, Matzen P, Munkholm P, Sørensen TIA: Increased risk of intestinal cancer in Crohn's disease: a meta-analysis of population-based cohort studies. Am J Gastroenterol 2005; 100:2724-2729.

4 Gillen CD, Andrews HA, Prior P, Allan RN: Crohn's disease and colorectal cancer. Gut 1994;35:651-655.

5 Ekbom A, Helmick C, Zack M, Adami HO: Ulcerative colitis and colorectal cancer: a population-based study. N Engl J Med 1990; 323:1228-1233.
6 Soetikno RM, Lin OS, Heidenreich PA, Young HS, Blackstone MO: Increased risk of colorectal neoplasia in patients with primary sclerosing cholangitis and ulcerative colitis: a meta-analysis. Gastrointest Endosc 2002; 56:48-54.

7 Eaden JA, Abrams KR, Mayberry JF: The risk of colorectal cancer in ulcerative colitis: a meta-analysis. Gut 2001;48:526-535. 
8 Lashner BA, Turner BC, Bostwick DG, Frank PH, Hanauer SB: Dysplasia and cancer complicating strictures in ulcerative colitis. Dig Dis Sci 1990;35:349-352.

-9 Velayos FS, Loftus EV, Jess T, Harmsen WS, Bida J, Zinsmeister AR, et al: Predictive and protective factors associated with colorectal cancer in ulcerative colitis: a case-control study. Gastroenterology 2006;130:19411949.

10 Lukas M: Inflammatory bowel disease as a risk factor for colorectal cancer. Dig Dis 2010;28:619-624.

11 Vogelstein B, Fearon ER, Hamilton SR, Kern SE, Preisinger AC, Leppert M, et al: Genetic alterations during colorectal-tumor development. N Engl J Med 1988;319:525-532.

-12 Nuako KW, Ahlquist DA, Mahoney DW, Schaid DJ, Siems DM, Lindor NM: Familial predisposition for colorectal cancer in chronic ulcerative colitis: a case-control study. Gastroenterology 1998;115:10791083.

13 Fearon ER, Vogelstein B: A genetic model for colorectal tumorigenesis. Cell 1990;61:759_ 767.

- 14 Tarmin L, Yin J, Harpaz N, Kozam M, Noordzij J, Antonio LB, et al: Adenomatous polyposis coli gene mutations in ulcerative colitis-associated dysplasias and cancers versus sporadic colon neoplasms. Cancer Res 1995;55:2035-2038.

-15 Fogt F, Vortmeyer AO, Goldman H, Giordano TJ, Merino MJ, Zhuang Z: Comparison of genetic alterations in colonic adenoma and ulcerative colitis-associated dysplasia and carcinoma. Hum Pathol 1998;29:131-136.

-16 Burmer GC, Rabinovitch PS, Haggitt RC, Crispin DA, Brentnall TA, Kolli VR, et al: Neoplastic progression in ulcerative colitis: histology, DNA content, and loss of a p53 allele. Gastroenterology 1992;103:1602-1610.

- 17 Hussain SP, Amstad P, Raja K, Ambs S, Nagashima M, Bennett WP, et al: Increased p53 mutation load in noncancerous colon tissue from ulcerative colitis: a cancer-prone chronic inflammatory disease. Cancer Res 2000;60:3333-3337.

-18 Brentnall TA, Crispin DA, Rabinovitch PS, Haggitt RC, Rubin CE, Stevens AC, et al: $\mathrm{Mu}-$ tations in the p53 gene: an early marker of neoplastic progression in ulcerative colitis. Gastroenterology 1994;107:369-378.

$\checkmark 19$ Xavier RJ, Podolsky DK: Unravelling the pathogenesis of inflammatory bowel disease. Nature 2007;448:427-434.

20 Hofseth LJ, Saito S, Hussain SP, Espey MG, Miranda KM, Araki Y, et al: Nitric oxide-induced cellular stress and p53 activation in chronic inflammation. Proc Natl Acad Sci USA 2003;100:143-148.

-21 Cravo ML, Albuquerque CM, Salazar de Sousa L, Glória LM, Chaves P, Dias Pereira A, et al: Microsatellite instability in non-neoplastic mucosa of patients with ulcerative colitis: effect of folate supplementation. Am J Gastroenterol 1998;93:2060-2064.
22 Itzkowitz SH: Molecular biology of dysplasia and cancer in inflammatory bowel disease. Gastroenterol Clin North Am 2006;35:553571.

23 Amit S, Ben-Neriah Y: NF-kappaB activation in cancer: a challenge for ubiquitination- and proteasome-based therapeutic approach. Semin Cancer Biol 2003;13:15-28.

24 Greten FR, Eckmann L, Greten TF, Park JM, Li ZW, Egan LJ, et al: IKKbeta links inflammation and tumorigenesis in a mouse model of colitis-associated cancer. Cell 2004;118: 285-296.

25 Rakoff-Nahoum S, Paglino J, Eslami-Varzaneh F, Edberg S, Medzhitov R: Recognition of commensal microflora by toll-like receptors is required for intestinal homeostasis. Cell 2004;118:229-241.

26 Becker C, Fantini MC, Schramm C, Lehr HA, Wirtz S, Nikolaev A, et al: TGF-beta suppresses tumor progression in colon cancer by inhibition of IL- 6 trans-signaling. Immunity 2004;21:491-501.

27 Chung Y-C, Chang Y-F: Significance of inflammatory cytokines in the progression of colorectal cancer. Hepatogastroenterology 2003;50:1910-1913.

28 Popivanova BK, Kitamura K, Wu Y, Kondo T, Kagaya T, Kaneko S, et al: Blocking TNFalpha in mice reduces colorectal carcinogenesis associated with chronic colitis. J Clin Invest 2008;118:560-570.

29 Kim YJ, Hong KS, Chung JW, Kim JH, Hahm KB: Prevention of colitis-associated carcinogenesis with infliximab. Cancer Prev Res (Phila) 2010;3:1314-1333.

-30 Hurwitz H, Fehrenbacher L, Novotny W, Cartwright T, Hainsworth J, Heim W, et al: Bevacizumab plus irinotecan, fluorouracil, and leucovorin for metastatic colorectal cancer. N Engl J Med 2004;350:2335-2342.

- 31 Costa C, Incio J, Soares R: Angiogenesis and chronic inflammation: cause or consequence? Angiogenesis 2007;10:149-166.

32 Yoo S-A, Kwok S-K, Kim W-U: Proinflammatory role of vascular endothelial growth factor in the pathogenesis of rheumatoid arthritis: prospects for therapeutic intervention. Mediators Inflamm 2008;2008:129873.

33 Scaldaferri F, Vetrano S, Sans M, Arena V, Straface G, Stigliano E, et al: VEGF-A links angiogenesis and inflammation in inflammatory bowel disease pathogenesis. Gastroenterology 2009;136:585-595.e5.

34 Waldner MJ, Wirtz S, Jefremow A, Warntjen M, Neufert C, Atreya R, et al: VEGF receptor signaling links inflammation and tumorigenesis in colitis-associated cancer. J Exp Med 2010;207:2855-2868.

35 Jänne PA, Mayer RJ: Chemoprevention of colorectal cancer. N Engl J Med 2000;342: 1960-1968.

36 Raju R, Cruz-Correa M: Chemoprevention of colorectal cancer. Dis Colon Rectum 2006; 49:113-124, discussion 124-125.
37 Velayos FS, Terdiman JP, Walsh JM: Effect of 5-aminosalicylate use on colorectal cancer and dysplasia risk: a systematic review and metaanalysis of observational studies. Am J Gastroenterol 2005;100:1345-1353.

38 Ishikawa TO, Herschman HR: Tumor formation in a mouse model of colitis-associated colon cancer does not require COX-1 or COX-2 expression. Carcinogenesis 2010;31: 729-736.

39 Collins PD, Mpofu C, Watson AJ, Rhodes JM: Strategies for detecting colon cancer and/or dysplasia in patients with inflammatory bowel disease. Cochrane Database Syst Rev 2006:CD000279.

-40 Rutter MD, Saunders BP, Wilkinson KH, Rumbles S, Schofield G, Kamm MA, et al: Cancer surveillance in longstanding ulcerative colitis: endoscopic appearances help predict cancer risk. Gut 2004;53:1813-1816.

-41 Bernstein CN, Shanahan F, Weinstein WM: Are we telling patients the truth about surveillance colonoscopy in ulcerative colitis? Lancet 1994;343:71-74.

42 Rubin CE, Haggitt RC, Burmer GC, Brentnall TA, Stevens AC, Levine DS, et al: DNA aneuploidy in colonic biopsies predicts future development of dysplasia in ulcerative colitis. Gastroenterology 1992;103:16111620.

-43 Blackstone MO, Riddell RH, Rogers BH, Levin B: Dysplasia-associated lesion or mass (DALM) detected by colonoscopy in longstanding ulcerative colitis: an indication for colectomy. Gastroenterology 1981;80:366374.

44 Ullman T, Odze R, Farraye FA: Diagnosis and management of dysplasia in patients with ulcerative colitis and Crohn's disease of the colon. Inflamm Bowel Dis 2009;15:630.

45 Hoffman A, Kagel C, Goetz M, Biesterfeld S, Galle PR, Neurath MF, et al: High definition colonoscopy (HD plus) with i-scan function allows to recognize and characterize flat neoplastic changes as precisely as chromoendoscopy. Gastrointest Endosc 2008; 67:AB125-AB125.

46 Kudo S, Tamura S, Nakajima T, Yamano H, Kusaka H, Watanabe H: Diagnosis of colorectal tumorous lesions by magnifying endoscopy. Gastrointest Endosc 1996;44:8-14.

47 Neumann H, Mönkemüller K, Günther C, Atreya R, Vieth M, Neurath MF: Advanced endoscopic imaging for diagnosis of Crohn's disease. Gastroenterol Res Pract 2012;2012: 301541.

48 Kiesslich R, Fritsch J, Holtmann M, Koehler $\mathrm{HH}$, Stolte M, Kanzler S, et al: Methylene blue-aided chromoendoscopy for the detection of intraepithelial neoplasia and colon cancer in ulcerative colitis. Gastroenterology $2003 ; 124: 880-888$.

49 Rutter MD, Saunders BP, Schofield G, Forbes A, Price AB, Talbot IC: Pancolonic indigo carmine dye spraying for the detection of dysplasia in ulcerative colitis. Gut 2004;53: 256-260. 
$50 \mathrm{Wu}$ L, Li P, Wu J, Cao Y, Gao F: The diagnostic accuracy of chromoendoscopy for dysplasia in ulcerative colitis: meta-analysis of six randomized controlled trials. Colorectal Dis 2012;14:416-420.

51 Pellisé M, López-Cerón M, Rodríguez de Miguel C, Jimeno M, Zabalza M, Ricart E, et al: Narrow-band imaging as an alternative to chromoendoscopy for the detection of dysplasia in long-standing inflammatory bowel disease: a prospective, randomized, crossover study. Gastrointest Endosc 2011;74: 840-848.

\$2 Hoffman A, Sar F, Goetz M, Tresch A, Mudter J, Biesterfeld S, et al: High definition colonoscopy combined with i-scan is superior in the detection of colorectal neoplasias compared with standard video colonoscopy: a prospective randomized controlled trial. Endoscopy 2010;42:827-833.

53 Kiesslich R: Confocal laser endoscopy for diagnosing intraepithelial neoplasias and colorectal cancer in vivo. Gastroenterology 2004;127:706-713.

54 Kiesslich R, Goetz M, Lammersdorf K, Schneider C, Burg J, Stolte M, et al: Chromoscopy-guided endomicroscopy increases the diagnostic yield of intraepithelial neoplasia in ulcerative colitis. Gastroenterology 2007;132:874-882.

-55 Hurlstone DP, Thomson M, Brown S, Tiffin N, Cross SS, Hunter MD: Confocal endomicroscopy in ulcerative colitis: differentiating dysplasia-associated lesional mass and adenoma-like mass. Clin Gastroenterol Hepatol 2007;5:1235-1241.
56 Foersch S, Kiesslich R, Waldner MJ, Delaney P, Galle PR, Neurath MF, et al: Molecular imaging of VEGF in gastrointestinal cancer in vivo using confocal laser endomicroscopy. Gut 2010;59:1046-1055.

57 Goetz M, Ziebart A, Foersch S, Vieth M, Waldner MJ, Delaney $\mathrm{P}$, et al: In vivo molecular imaging of colorectal cancer with confocal endomicroscopy by targeting epidermal growth factor receptor. Gastroenterology 2010;138:435-446.

58 Neurath MF, Kiesslich R: Molecular detection of CD44v6 on aberrant crypt foci by confocal laser endoscopy. Endoscopy 2010; 42(suppl 2):E314-E315.

59 Eaden J, Abrams K, Ekbom A, Jackson E, Mayberry J: Colorectal cancer prevention in ulcerative colitis: a case-control study. Aliment Pharmacol Ther 2000;14:145-153.

-60 Lindberg B, Persson B, Veress B, IngelmanSundberg H, Granqvist S: Twenty years' colonoscopic surveillance of patients with ulcerative colitis: detection of dysplastic and malignant transformation. Scand J Gastroenterol 1996;31:1195-1204.

61 Pardi DS, Loftus EV, Kremers WK, Keach J, Lindor KD: Ursodeoxycholic acid as a chemopreventive agent in patients with ulcerative colitis and primary sclerosing cholangitis. Gastroenterology 2003;124:889-893.

62 Tung BY, Emond MJ, Haggitt RC, Bronner MP, Kimmey MB, Kowdley KV, et al: Ursodiol use is associated with lower prevalence of colonic neoplasia in patients with ulcerative colitis and primary sclerosing cholangitis. Ann Intern Med 2001;134:89-95.
63 Ikegami T, Matsuzaki Y: Ursodeoxycholic acid: mechanism of action and novel clinical applications. Hepatol Res 2008;38:123-131.

-64 Eaton JE, Silveira MG, Pardi DS, Sinakos E, Kowdley KV, Luketic VAC, et al: High-dose ursodeoxycholic acid is associated with the development of colorectal neoplasia in patients with ulcerative colitis and primary sclerosing cholangitis. Am J Gastroenterol 2011;106:1638-1645.

-65 Caspersen S, Elkjaer M, Riis L, Pedersen N, Mortensen C, Jess T, et al: Infliximab for inflammatory bowel disease in Denmark 1999-2005: clinical outcome and follow-up evaluation of malignancy and mortality. Clin Gastroenterol Hepatol 2008;6:12121217, quiz 1176.

66 Baumgart DC: Endoscopic surveillance in Crohn's disease and ulcerative colitis: who needs what and when? Dig Dis 2011;29(suppl 1):32-35.

67 Ullman TA, Itzkowitz SH: Intestinal inflammation and cancer. Gastroenterology 2011; 140:1807-1816.

68 Galon J, Costes A, Sanchez-Cabo F: Type, density, and location of immune cells within human colorectal tumors predict clinical outcome. Science 2006;313:1960-1964.

69 Baumgart DC: Recognition and appropriate management of dysplasia-associated lesions or masses in inflammatory bowel disease experience does matter. Gastrointest Endosc 2007;66:530-532.

70 Farraye FA, Odze RD, Eaden J, Itzkowitz SH: AGA technical review on the diagnosis and management of colorectal neoplasia in inflammatory bowel disease. Gastroenterology $2010 ; 138: 746-774$. 